\title{
Enzymatic degradation of graphene quantum dots by human peroxidases
}

Cristina Martín, Gong Jun, Rachel Schurhammer, Giacomo Reina, Peng Chen, Alberto Bianco, ${ }^{*}$ and Cécilia Ménard-Moyon*

Dr. Cristina Martín, Dr. Giacomo Reina, Dr. Alberto Bianco, Dr. Cécilia Ménard-Moyon University of Strasbourg, CNRS, Immunology, Immunopathology and Therapeutic Chemistry, UPR 3572, 67000 Strasbourg, France.

M. Gong Jun, Prof. Peng Chen

School of Chemical \& Biomedical Engineering, 62 Nanyang Drive, Singapore 637459.

Prof. Rachel Schurhammer

Laboratoire de Modélisation et Simulations Moléculaires \& Laboratoire de Chimie Moléculaire de l'Etat Solide (UMR 7140 CNRS), Université de Strasbourg, 1 rue Blaise Pascal, 67081 Strasbourg, France.

E-mail: a.bianco@ibmc-cnrs.unistra.fr, c.menard@ibmc-cnrs.unistra.fr

Keywords: Carbon materials, fluorescence, myeloperoxidase, eosinophil peroxidase, molecular dynamics simulations

Carbon-based nanomaterials have demonstrated to be potent candidates for biomedical applications. Recently, graphene quantum dots (GQDs) have emerged as an attractive tool for bioimaging, biosensing and therapy. Hence, studying their biodegradability in living systems is essential to speed up the translation towards real clinical innovations. Here, the enzymatic degradation of GQDs using human myeloperoxidase and eosinophil peroxidase has been investigated. Transmission electron microscopy, fluorescence and Raman spectroscopy were used to evaluate the biodegradation of GQDs. Signs of degradation by both enzymes were observed already after a few hours of incubation with each enzyme, being more evident after a couple of days of treatment. Molecular dynamics simulations showed intimate interactions between the enzymes and the GQDs. The conformation of both peroxidases was slightly altered to favor the interactions, while the GQD sheets distorted a little to adapt to the surface of the enzymes. The biodegradability of the GQDs ensures their real potential in the practical biomedical applications. 


\section{Introduction}

Graphene quantum dots are zero-dimensional carbon nanomaterials resembling the crystalline structure of single or a-few layered graphene with a lateral size of a few nm and a circular or ellipsoidal shape ${ }^{[1,2]}$ Due to quantum confinement, edge effects, chemical modification and heteroatom doping, GQDs display remarkable chemicophysical properties, attracting increased attention of scientists in different fields spanning from physics and chemistry to materials science and biology. ${ }^{[3]}$ In addition, GQDs have highly tunable photoluminescence, good photostability, and sometimes unusual multi-photon excitation property, which make them desirable candidates for a wide range of applications such as bioimaging, optical sensing, photocatalysis, and optoelectronics. ${ }^{[4-6]}$

Owing to its molecular size and photostability, GQDs are advantageous for real-time tracking of cells or cell receptors. ${ }^{[1,7]}$ For instance, Zheng et al. synthesized insulin-conjugated GQDs for specific labeling and dynamic tracking of insulin receptors in 3T3-L1 adipocytes. ${ }^{[8]}$ Kumawat et al. designed a one-step microwave-assisted method for the synthesis of biocompatible GQDs using mango leaves as a carbon precursor. ${ }^{[9]}$ They demonstrated how the surface modification of GQDs can tune the fluorescence emission accomplishing its potential toward in vivo bioimaging. In addition, nitrogen-doped GQDs obtained by hydrothermal treatment of graphene oxide in the presence of ammonia, are promising candidates for diagnosis of diseases since they were internalized by HeLa cells showing bright luminescence and high biocompatibility. ${ }^{[10]}$

Thanks to their biocompatibility, unique structure and properties, GQDs have also been used as multifunctional nanoplatforms for anticancer therapy while allowing simultaneous visual monitoring. ${ }^{[11,12]}$ For instance, utilizing their capacity to produce ${ }^{1} \mathrm{O}_{2}$ in the visible light GQDs can induce tumor ablation in mice by photodynamic therapy. ${ }^{[13]}$ In another example GQDs were synthesized using withered leaves by one-pot hydrothermal synthesis. ${ }^{[14]}$ These GQDs 
have photothermal and photodynamic properties, exerting a potent therapeutic activity to breast cancer cells. ${ }^{[14]}$

Beside demonstrating that GQDs are biocompatible, it is also fundamental to assess their biodegradability in living systems. To the best of our knowledge, the biodegradation of GQDs has not been investigated yet. Only few works reported the degradation of carbon dots, which are quasi-spherical amorphous or crystalline nanoparticles with a diameter usually less than $10 \mathrm{~nm}$, and they are distinct to GQDs in terms of morphology and properties. ${ }^{[2,3]}$ The carbon dots can be completely degraded into $\mathrm{CO}_{2}, \mathrm{CO}$, and $\mathrm{H}_{2} \mathrm{O}$ under visible light for 30 days. ${ }^{[15]} \mathrm{In}$ addition, Srivastava et al. ${ }^{[16]}$ have recently demonstrated that carbon dots undergo peroxidecatalyzed degradation in the presence of lipase.

Here, we have studied the degradation of GQDs using human myeloperoxidase (MPO) and eosinophil peroxidase (EPO), enzymes that are expressed in activated neutrophils and eosinophil granulocytes, respectively. The effective degradation ability of these enzymes towards other nanomaterials such as carbon nanotubes, ${ }^{[17,18]}$ graphene oxide sheets, ${ }^{[19]}$ or single and few-layer graphene sheets ${ }^{[20,21]}$ has been previously demonstrated.

\section{Results and Discussion}

The GQDs used in this study were synthesized by hydrothermal treatment of 1,3,6trinitropyrene in an alkaline aqueous solution at $200{ }^{\circ} \mathrm{C}$ as previously reported (see experimental details in Supporting Information). ${ }^{[22]}$ This process performed in $\mathrm{NaOH}$ aqueous solution allowed to obtain GQDs functionalized with hydroxyl groups, most likely located at the edges. The GQDs have a lateral size of $\sim 3.5 \mathrm{~nm}$ and contain 1-2 layers in average. ${ }^{[22]}$ They are negatively charged with a zeta potential of $-31.4 \mathrm{mV}(\mathrm{SD}+/-6.02 \mathrm{mV})$. In order to carry out the degradation experiments, both MPO and EPO were mixed with the GQDs in phosphate-buffered saline (PBS) and the solutions were maintained at $37^{\circ} \mathrm{C}$ for $40 \mathrm{~h}$ 
(hereafter the treated samples are named " $T$ "). ${ }^{[18,19]}$ We also performed control experiments (named “C”) by incubating the GQDs with only hydrogen peroxide in order to see if it could alter their structure. Different aliquots of the different samples were collected at incubation time points of 5, 10, 20, and $40 \mathrm{~h}$ for further analyses. The first evidence of degradation was the observed decrease of the color intensity of the dispersions over time. The change occurred not only for the control samples due to dilution, since aliquots $(100 \mu \mathrm{L})$ were collected at each incubation time point for further analyses and the same volume was replenished with fresh buffer, but clearly for the enzyme-treated samples due to degradation (Figure S1).

We further analyzed the fluorescence of the samples at different time points. The maximum emission of the GQDs was reached at $541 \mathrm{~nm}$ upon excitation at $488 \mathrm{~nm}$ (Figure S2). The concentration of the samples was calculated from a calibration curve of different GQD dilutions (Figure S3). Figure 1 shows a clear decrease in the fluorescence intensity of each enzyme-treated sample compared to its respective control (designated as 100\%) after different incubation time points. In comparison, the fluorescence of the samples was not altered in the presence of only $\mathrm{H}_{2} \mathrm{O}_{2}$. Indeed, the fluorescence of the control sample $\left(\mathrm{H}_{2} \mathrm{O}_{2}\right.$-treated GQDs) was stable upon storage for 3 days in the dark. Moreover, it is necessary to highlight that the fluorescence was not quenched by the enzymes as the fluorescence of the GQDs treated only with MPO or EPO (without $\mathrm{H}_{2} \mathrm{O}_{2}$ ) was similar to that of the GQDs (data not shown). Taken together, these data are indicative of a progressive decrease in the concentration of the GQDs over time caused by the enzymatic degradation. The decay in the fluorescence intensity was more evident during the first $5 \mathrm{~h}$ of incubation compared to the rest of the time intervals. This result may be explained by the high amount of oxygenated groups present on the surface of the GQDs, which make them highly water-dispersible and favor the interactions with the enzyme. We hypothesize that after degrading the defective parts of the GQDs, the enzyme 
started degrading the more graphitic areas, which could explain why the speed of degradation process was slowed down after $5 \mathrm{~h}$.

An agarose gel electrophoresis was then performed to evaluate the UV signal of the samples after different periods of enzymatic degradation (Figure 2). Aliquots of the controls and enzyme-treated GQDs were mixed with 6X Orange DNA loading dye to increase the viscosity of the samples and allow GQD staining. The mixtures were added to $1 \%$ agarose gel followed by electrophoresis. The gels were then visualized under UV light. The signal of each enzyme-treated sample was normalized to the signal of its respective control and subtracted from the background signal. After incubating the GQDs with both MPO and EPO, the signal intensity decreased over time (Figure $2 b$ and $d$ ), indicating a lower concentration of the GQDs due to degradation. These results are in agreement with the fluorescence data showing that the concentration drastically decreased over time, not only for the control samples due to dilution, but also for the enzyme-treated samples, and especially during the first $5 \mathrm{~h}$ of the treatment, confirming the degradation capacity of the GQDs using MPO or EPO. The electrophoresis experiment rules out the possibility that fluorescence decrease is not due to oxidation of GQDs by the enzymes. To corroborate that the decrease in the signal intensity was a direct consequence of the lower GQD concentration due to degradation, we also tested two lower concentrated suspensions of GQDs (without any enzyme treatment). After running the gel in the same conditions, both signals decreased proportionally compared to the one of the initial GQD concentration (Figure S4), meaning that a lower concentration of GQDs correspond to a lower UV signal.

To further support the degradation of the GQDs, we analyzed the samples using spectroscopic and high resolution microscopic techniques. First, Raman spectroscopy was used to monitor the evolution of the $\mathrm{D}$ band $\left(\sim 1375 \mathrm{~cm}^{-1}\right)$ and $\mathrm{G}$ peak $\left(\sim 1600 \mathrm{~cm}^{-1}\right)$, corresponding to disordered carbon and to the $\mathrm{sp}^{2}$ tangential mode, respectively (Figure 3). ${ }^{[23]}$ During the 
course of the treatment, both bands became less intense, indicating of a progressive loss of the GQD structure. ${ }^{[17,20]}$ Indeed, the intensities of both D and G peaks were significantly reduced already after $5 \mathrm{~h}$ of enzymatic treatment compared to the control sample $\left(\mathrm{H}_{2} \mathrm{O}_{2}\right.$-treated GQDs for $40 \mathrm{~h}$ ), which was similar to the enzyme-treated sample at $\mathrm{t}=0 \mathrm{~h}$ confirming that $\mathrm{H}_{2} \mathrm{O}_{2}$ does not alter the GQD structure (Figure S5). The two bands almost disappeared after $40 \mathrm{~h}$ of incubation for both enzymatic treatments. These results confirm the gradual loss of the GQD structure due to degradation by the enzyme and not by $\mathrm{H}_{2} \mathrm{O}_{2}$ alone.

To investigate the changes in the structure and the morphology of the GQDs after incubation with both MPO and EPO, high resolution transmission electron microscopy (HRTEM) analyses were carried out. For this purpose, aliquots of the enzyme-treated GQD samples were filtered using centrifugal filter units (10000 MWCO) in order to remove the enzyme before deposition onto the TEM grids and subsequent washing with water to remove salts. We observed by HRTEM the remaining GQDs that were not completely degraded by the enzymes. We clearly noticed a partial loss of the lattice structure, confirming again the degradation capacity of MPO and EPO (Figure 4 and S6). This evidence was found in most of the GQDs present on the HRTEM grids. This is certainly due to the process of oxidation and consequent degradation of the GQDs, leading to less graphitic structures (as indicated by the white arrows in Figure 4) compared to the control samples (Figure S7 and S8). The remaining graphitic parts of the GQDs exhibited well-defined crystal lattices with a graphitic in-plane lattice spacing of $0.23 \mathrm{~nm} .{ }^{[2]}$ These results are in agreement with previous reports on other types of carbon nanomaterials showing a partial degradation mainly due to the rather short duration of the study, which is limited by the instability of the enzymes in test tube experiments. ${ }^{[20]}$

Finally, we studied the interactions between the GQDs and MPO or EPO i) experimentally by polyacrylamide gel electrophoresis, and ii) theoretically by molecular dynamics (MD) 
simulations. To perform the electrophoresis analysis, MPO and EPO were incubated with the GQDs in a 1:2 weight ratio at room temperature under non-reducing conditions for $24 \mathrm{~h}$ before the electrophoresis. The analysis showed a decrease in the intensity of the bands corresponding to the enzymes incubated with the GQDs compared to MPO and EPO alone (Figure S9). Values of $90.6 \pm 4.8 \%$ and $92.6 \pm 2.5 \%$ of band intensities were obtained for MPO/GQDs and EPO/GQDs, respectively, assuming 100\% of band intensity for MPO alone or EPO alone used as controls. This result pointed out that MPO and EPO bind to the GQDs, reducing the amount of protein migrating into the gel. The binding of the enzymes is possible through electrostatic interactions between the cationic enzymes and the negatively charged GQDs bearing mainly hydroxyl groups.

In the attempt to better understand the interactions between the GQDs and the enzymes, we performed molecular dynamics (MD) simulations. MPO and EPO were surrounded by GQDs of two types: i) GQDs functionalized at the edges with hydroxyl groups (as synthesized in this study), and ii) GQDs bearing instead hydrogen atoms at the edges (H-GQDs). The GQDs were mimicked as a singled-layered graphene structure of about $40 \AA$ in diameter to reduce computational costs. The systems were simulated with either 4 or 20 GQDs. In the latter case spontaneous aggregation of the sheets forming aggregates of around five layers was observed. MD results showed no particular differences regarding adsorption on the surface of the enzymes for both systems (4 vs. 20 GQDs). The kinds of GQDs were dispersed randomly around the enzymes. H-GQDs were used as control to assess the effect of the hydroxyl groups on the interactions with the enzymes. The MD simulations of MPO and EPO were run according to the procedure described in SI. The initial and final snapshots of the simulated systems are presented in Figure S10. The final views obtained after 100 ns of dynamics (Figure 5a and S11) clearly show the aptitude of the H-GQDs and GQDs to adsorb on the surface of both MPO and EPO. The molecular modeling simulation demonstrates the possible 
interaction sites of both types of GQDs with the peroxidases. The simulation suggests that one enzyme may interact directly with up to six GQDs. Once the GQDs adsorb on the surface of the peroxidase, they remain at the same averaged position during all the simulation. The excess GQDs interact via $\pi-\pi$ interactions with the GQDs adsorbed on the peroxidase and thus form aggregates of a few layers around the enzyme. We noticed that the active site of both enzymes seems not to be accessible to both types of GQDs.

The interaction energies calculated between the whole enzyme (MPO or EPO) and each type of GQDs during the last $10 \mathrm{~ns}$ of dynamics are given in Table S2. The interaction energies of the GQDs adsorbed on the enzymes are in the same order of magnitude for MPO and EPO, ranging from $\sim-300$ to $-100 \mathrm{kcal} \cdot \mathrm{mol}^{-1}$. The interactions between the enzymes and the GQDs are higher compared to the H-GQDs (the difference is $\sim 50 \mathrm{kcal} \cdot \mathrm{mol}^{-1}$ ). This distinction is due to the electrostatic representation of both GQDs as the H-GQDs are neutral (atomic charges: $q\left(H_{C}\right)=+0.16$ e and $q\left(C_{H}\right)=-0.34$ e), while the edges are polar for the GQDs due to the presence of the hydroxyl groups $\left(\mathrm{q}\left(\mathrm{H}_{\mathrm{O}}\right)=+0.34\right.$ e and $\left.\mathrm{q}\left(\mathrm{O}_{\mathrm{H}}\right)=-0.44 \mathrm{e}\right)$. These results highlight the crucial role of the functional groups at the GQD edges on the interactions with both peroxidases. The large interaction energy differences between the various GQDs around the enzymes clearly indicate that some positions are preferred over others. Indeed, the extreme positions of MPO are favored for strong interactions with the GQDs for the 4 and 20 H-GQD and GQD systems (Figure S11). Interestingly, the amino acid chains at these positions are quite mobile and become flattened to perfectly fit with the surface of the GQDs. In the case of EPO, there are many favored positions for GQD adsorption around the enzyme, four of them lead to the same order of interaction energies between the H-GQDs/GQDs and the peroxidase (Figure 5b, S11, S12, and Table S2). We also analyzed the nature and type of amino acids in interaction with the surface of the enzymes during the last $10 \mathrm{~ns}$ of dynamics. We considered two groups of amino acids: i) charged amino acids (Arg, Lys, His) and ii) 
aromatic ones (Phe, Trp, Tyr) assuming that these two groups may interact in a privileged way with the GQDs through electrostatic interactions as well as tangential cation- $\pi$ and $\pi$ stacking (Figure 5, S11, and S13). The proportion of amino acids interacting with the $\mathrm{H}$ GQDs/GQDs among the total and accessible number of amino acids are given in Table S3. Charged amino acids are more accessible in both peroxidases compared to aromatic amino acids, mainly due to the high number of arginine residues present on the enzyme surface. The interactions between the H-GQDs/GQDs and MPO/EPO are mainly driven by cation- $\pi$ interactions between the guanidinium moiety of Arg and the graphitic surface of the GQDs. In addition, cation- $\pi$ and $\pi-\pi$ interactions are also present with Lys/His and Phe/Trp/Tyr, respectively.

We finally performed root-mean-square-deviation (RMSD) calculations taking as a reference the final structure of the free enzymes simulated during $100 \mathrm{~ns}$ in water. Superposed structures and RMSD values are given in Figure S14. RMSD of MPO or EPO in water without or with the GQDs (or H-GQDs) varied from 4.2 to 5.6. The values were similar or higher for the system with 4 GQDs compared to 20 for MPO and EPO, respectively. These results indicated that the surrounding GQDs may somehow constrain the whole structure of the peroxidases, in particular EPO. The tertiary structure of both MPO and EPO remained practically unchanged during the MD simulation, and the final structure of the enzyme backbones taken from the different simulations (with or without GQDs) were similar. However, accessible amino acids that may interact with some GQDs, were modified during the simulation. The conformation of the enzymes was changed while interacting with the GQDs to favor interactions. In addition, the shape of the GQDs was also modified to adapt to the surface of the enzyme (Figure 5 and S11). Flexibility, distortion, and overall curvature modifications of GQDs and carbon dots have already been reported by MD studies. ${ }^{[24,25]}$ The 
accumulation of GQDs around the peroxidases led to the formation of sandwich-type aggregates that also exist in pure solutions (i.e. without the enzymes).

\section{Conclusion}

Both MPO and EPO were found to significantly degrade the GQDs in the presence of $\mathrm{H}_{2} \mathrm{O}_{2}$ and in the timeframe of the designed experiments $(40 \mathrm{~h})$. The biodegradation of the GQDs was confirmed by HRTEM, fluorescence and Raman spectroscopy, observing signs of degradation already after $5 \mathrm{~h}$ of incubation with the enzymes, and being very evident after $40 \mathrm{~h}$ of treatment. Moreover, the interactions of both enzymes with the GQDs were thoroughly investigated by molecular dynamics simulations. The conformation of MPO and EPO was slightly modified to favor the interactions with the GQDs, while the sheets were a little distorted to adapt to the surface of the enzymes. Overall, our study shows the first biodegradability evidence of GQDs, which emphasizes their real potential for biomedical applications and offers great opportunities in nanomedicine.

\section{Experimental Section}

The detailed information of materials, synthesis of GQDs, degradation experiments, characterization techniques and computational methods can be found in the Supporting Information.

\section{Supporting Information}

Supporting Information is available from the Wiley Online Library or from the author.

\section{Acknowledgements}

This work was supported by the Centre National de la Recherche Scientifique (CNRS) and by the International Center for Frontier Research in Chemistry (icFRC). The authors gratefully 
acknowledge financial support from EU GRAPHENE Flagship project (no. 785219) and from the Agence Nationale de la Recherche (ANR) through the LabEx project Chemistry of Complex Systems (ANR-10-LABX-0026_CSC), AcRF tier 2 grant (MOE2017-T2-2-005) from Ministry of Education of Singapore and AME-IRG grant (AMEIRG18-0016) from Agency for Science, Technology and Research of Singapore. The authors wish to thank Cathy Royer and Valérie Demais for help with TEM analyses at the 'Plateforme Imagerie in vitro' at the Center of Neurochemistry (Strasbourg, France). Dris Ihiawakrim and Belén Ballesteros are gratefully acknowledged for fruitful discussion.

\section{References}

[1] M. Bacon, S. J. Bradley, T. Nann, Part. Part. Syst. Charact. 2014, 31, 415.

[2] X. T. Zheng, A. Ananthanarayanan, K. Q. Luo, P. Chen, Small 2015, 11, 1620.

[3] Y. Yan, J. Gong, J. Chen, Z. Zeng, W. Huang, K. Pu, J. Liu, P. Chen, Adv. Mater. 2019, 31, 1808283.

[4] S.-J. Jeon, T.-W. Kang, J.-M. Ju, M.-J. Kim, J. H. Park, F. Raza, J. Han, H.-R. Lee, J.H. Kim, Adv. Funct. Mater. 2016, 26, 8211.

[5] A. Martín-Pacheco, A. E. Del Río Castillo, C. Martín, M. A. Herrero, S. Merino, J. L. García Fierro, E. Díez-Barra, E. Vázquez, ACS Appl. Mater. Interfaces 2018, 10, 18192.

[6] C. Shen, S. Ge, Y. Pang, F. Xi, J. Liu, X. Dong, P. Chen, J. Mater. Chem. B 2017, 5, 6593.

[7] J. Chen, A. Than, N. Li, A. Ananthanarayanan, X. Zheng, F. Xi, J. Liu, J. Tian, P. Chen, FlatChem 2017, 5, 25.

[8] X. T. Zheng, A. Than, A. Ananthanaraya, D.-H. Kim, P. Chen, ACS Nano 2013, 7, 6278. 
[9] M. K. Kumawat, M. Thakur, R. B. Gurung, R. Srivastava, ACS Sustain. Chem. Eng. 2017, 5, 1382.

[10] C. Hu, Y. Liu, Y. Yang, J. Cui, Z. Huang, Y. Wang, L. Yang, H. Wang, Y. Xiao, J. Rong, J. Mater. Chem. B 2013, 1, 39.

[11] D. Iannazzo, I. Ziccarelli, A. Pistone, J. Mater. Chem. B 2017, 5, 6471.

[12] K. Li, W. Liu, Y. Ni, D. Li, D. Lin, Z. Su, G. Wei, J. Mater. Chem. B 2017, 5, 4811.

[13] J. Ge, M. Lan, B. Zhou, W. Liu, L. Guo, H. Wang, Q. Jia, G. Niu, X. Huang, H. Zhou, X. Meng, P. Wang, C.-S. Lee, W. Zhang, X. Han, Nat. Commun. 2014, 5, 4596.

[14] M. Thakur, K. Kumawat, R. Srivastava, RSC Adv. 2017, 7, 5251.

[15] H. Li, J. Huang, Y. Song, M. Zhang, H. Wang, F. Lu, H. Huang, Y. Liu, X. Dai, Z. Gu, Z. Yang, R. Zhou, Z. Kang, ACS Appl. Mater. Interfaces 2018, 10, 26936.

[16] I. Srivastava, D. Sar, P. Mukherjee, A. S. Schwartz-Duval, Z. Huang, C. Jaramillo, A. Civantos, I. Tripathi, J. P. Allain, R. Bhargava, D. Pan, Nanoscale 2019, 11, 8226.

[17] V. E. Kagan, N. V. Konduru, W. Feng, B. L. Allen, J. Conroy, Y. Volkov, I. I. Vlasova, N. A. Belikova, N. Yanamala, A. Kapralov, Y. Y. Tyurina, J. Shi, E. R. Kisin, A. R. Murray, J. Franks, D. Stolz, P. Gou, J. Klein-Seetharaman, B. Fadeel, A. Star, A. A. Shvedova, Nat. Nanotechnol. 2010, 5, 354.

[18] F. T. Andón, A. A. Kapralov, N. Yanamala, W. Feng, A. Baygan, B. J. Chambers, K. Hultenby, F. Ye, M. S. Toprak, B. D. Brandner, A. Fornara, J. Klein-Seetharaman, G. P. Kotchey, A. Star, A. A. Shvedova, B. Fadeel, V. E. Kagan, Small 2013, 9, 2721.

[19] R. Kurapati, J. Russier, M. A. Squillaci, E. Treossi, C. Ménard-Moyon, A. E. Del RioCastillo, E. Vazquez, P. Samorì, V. Palermo, A. Bianco, Small 2015, 11, 3985.

[20] R. Kurapati, S. P. Mukherjee, C. Martín, G. Bepete, E. Vázquez, A. Pénicaud, B. Fadeel, A. Bianco, Angew. Chemie Int. Ed. 2018, 57, 11722.

[21] C. Martín, K. Kostarelos, M. Prato, A. Bianco, Chem. Commun. 2019, 55, 5540. 
[22] L. Wang, Y. Wang, T. Xu, H. Liao, C. Yao, Y. Liu, Z. Li, Z. Chen, D. Pan, L. Sun, M. Wu, Nat. Commun. 2014, 5, 5357.

[23] A. C. Ferrari, Solid State Commun. 2007, 143, 47.

[24] P. Elvati, E. Baumeister, A. Violi, $R S C A d v .2017$, 7, 17704.

[25] M. Paloncýová, M. Langer, M. Otyepka, J. Chem. Theory Comput. 2018, 14, 2076.
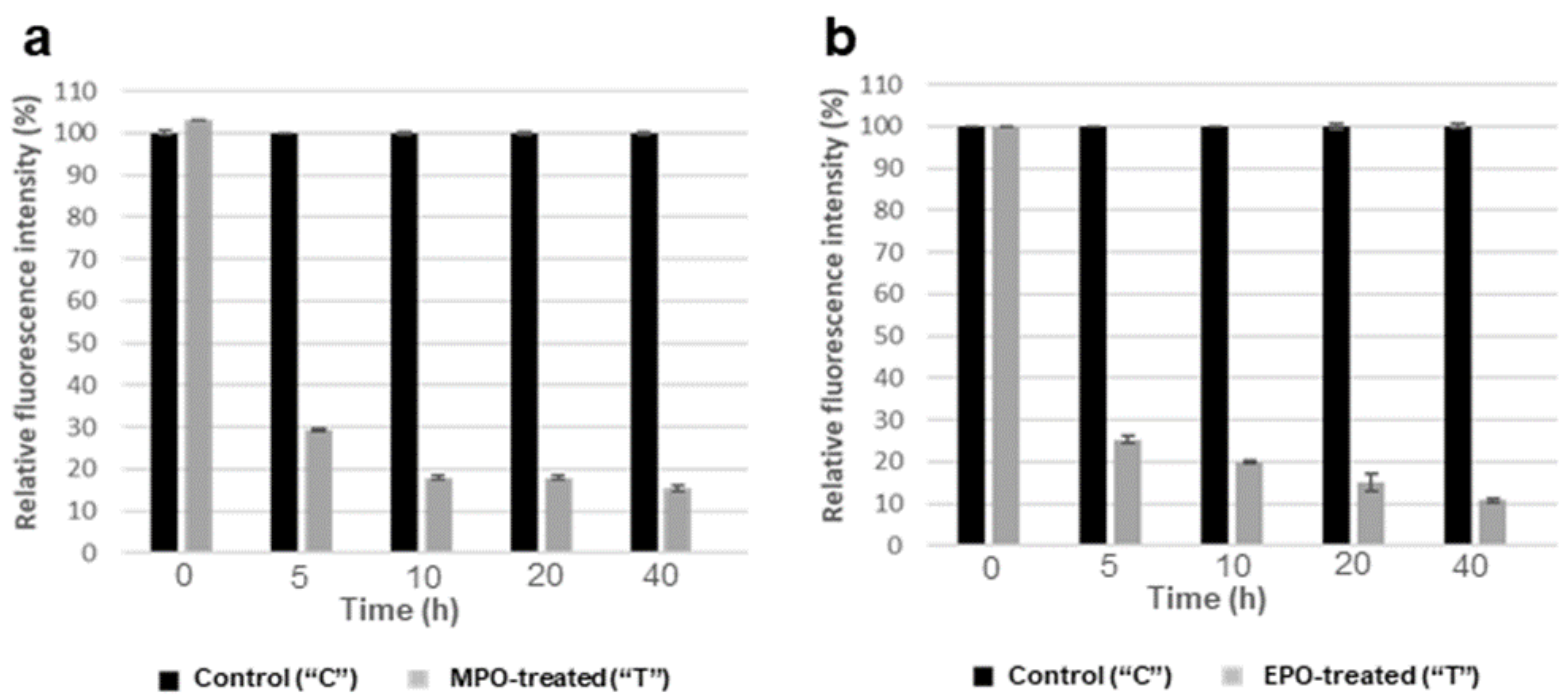

Figure 1. Fluorescence intensity of MPO-treated (a) and EPO-treated (b) GQDs ("T") and their respective control samples $\left(\mathrm{H}_{2} \mathrm{O}_{2}\right.$-treated GQDs " $\mathrm{C}$ ") at different incubation time points. The measurements were repeated three times. 

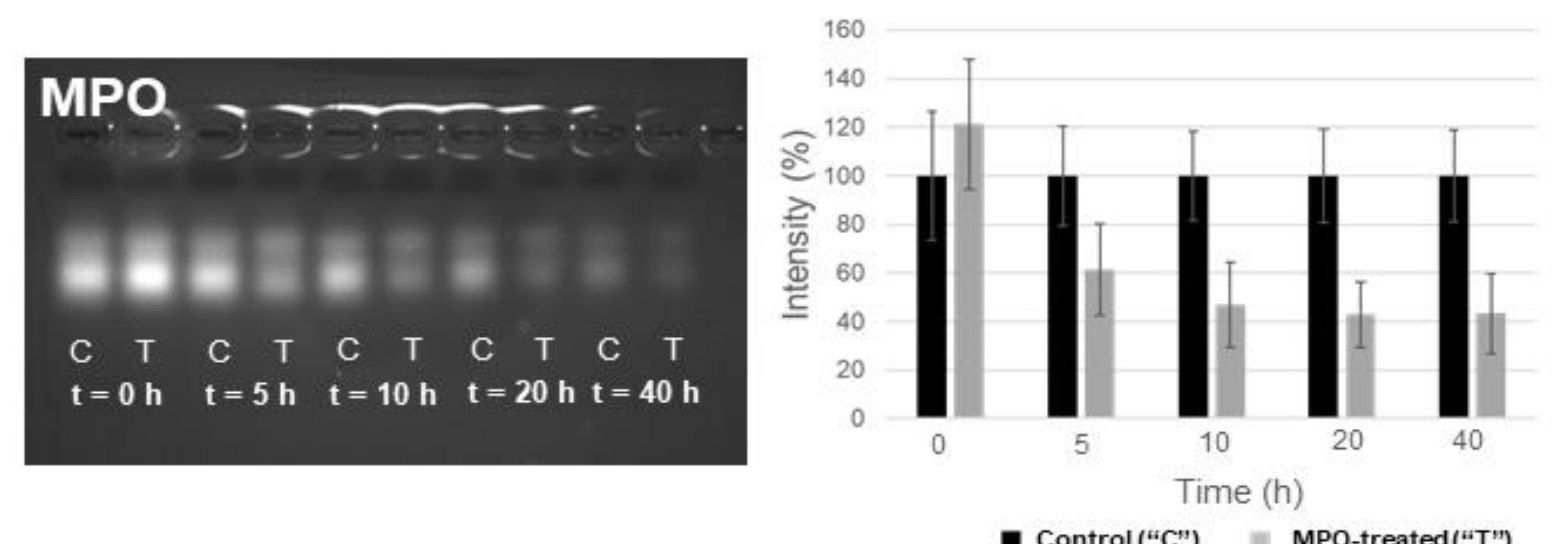

C

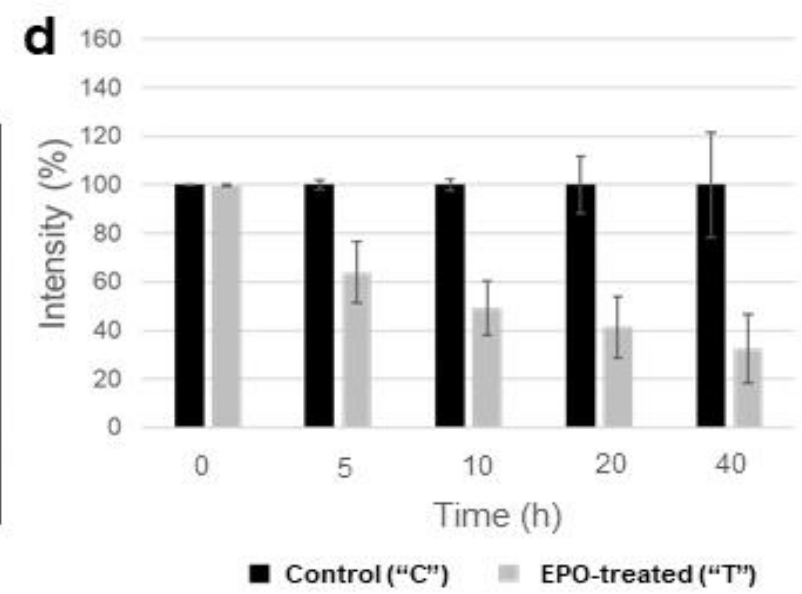

Figure 2. Images of the gel electrophoresis of GQDs treated ("T") with MPO (a) and EPO (c) and their respective controls $\left(\mathrm{H}_{2} \mathrm{O}_{2}\right.$-treated GQDs " $\mathrm{C}$ ") at different incubation time points. Right panels represent the intensity signal (\%) of the GQDs treated with MPO (b) and EPO (d) and their respective controls at different incubation time points.

a

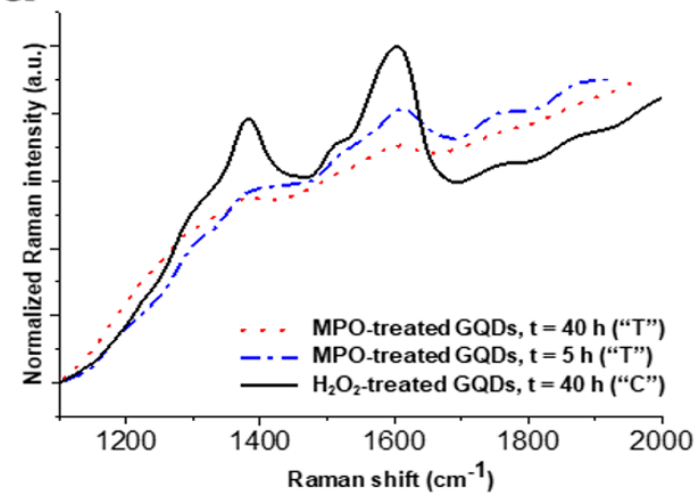

b

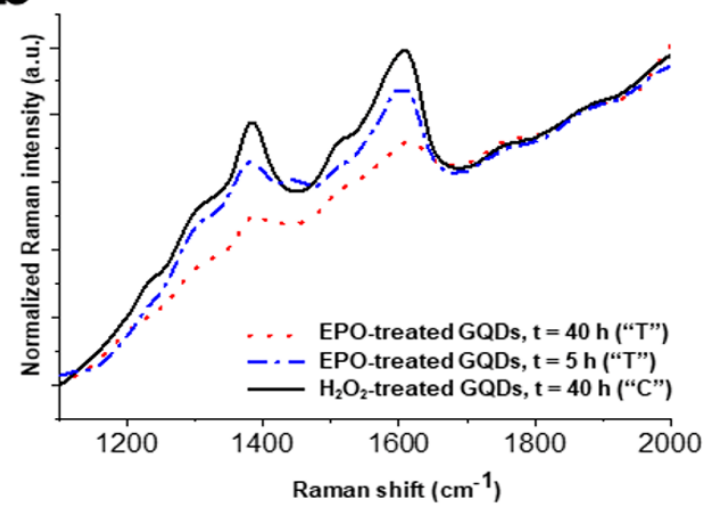

Figure 3. Normalized Raman average spectra of MPO- (a) and EPO-treated (b) samples ("T") after 5 and $40 \mathrm{~h}$ of incubation. Control samples ("C") were treated with $\mathrm{H}_{2} \mathrm{O}_{2}$ only ( $\mathrm{t}=40 \mathrm{~h}$ ). At least 30 different measurements were performed in several sample locations. 

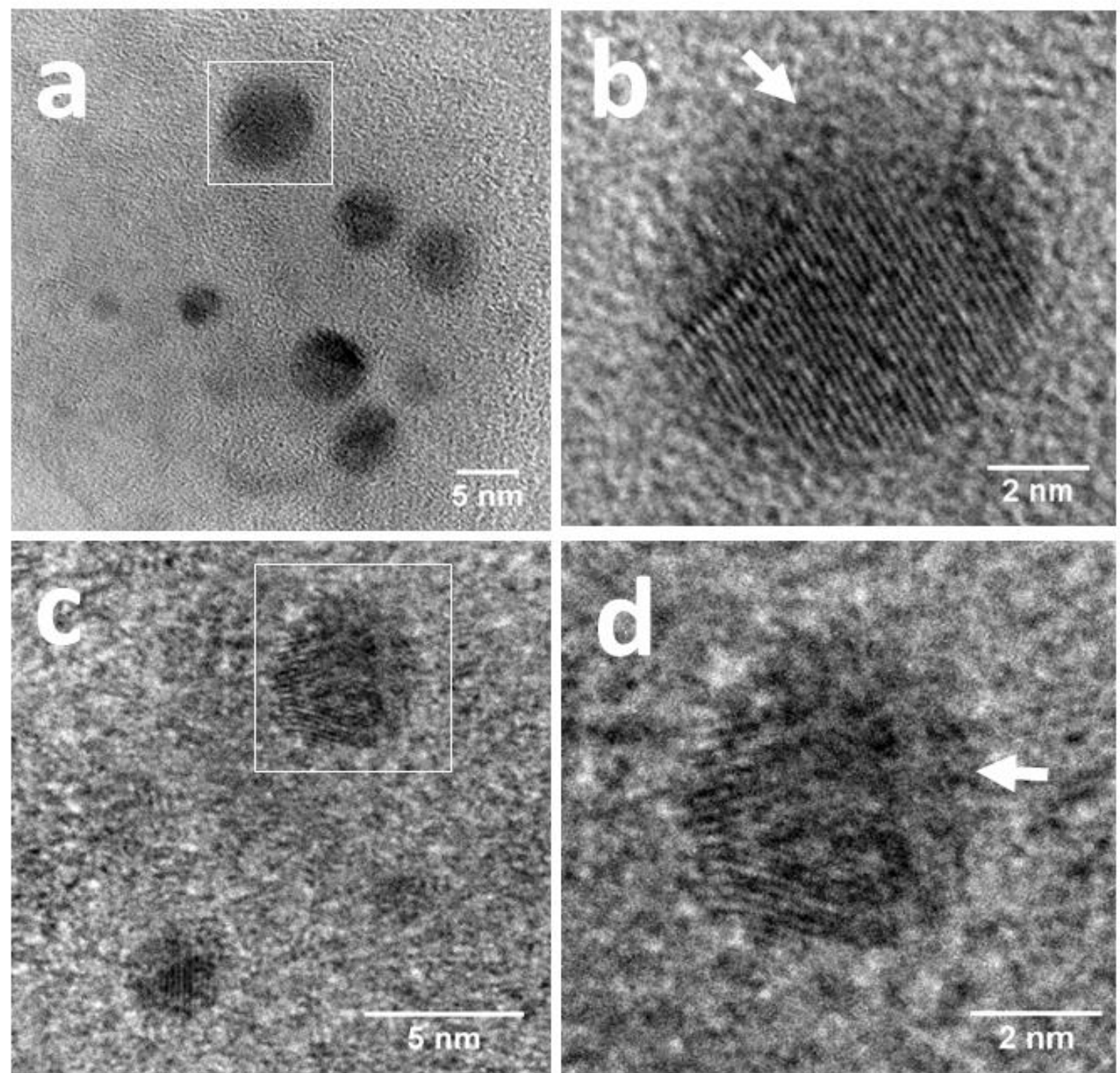

Figure 4. HRTEM images of the enzyme-treated GQDs after $40 \mathrm{~h}$ of incubation. a) MPOtreated GQDs (white square traces the inset displayed in b); c) EPO-treated GQDs (white square traces the inset displayed in d). White arrows indicate damaged structure of GQDs. 
a
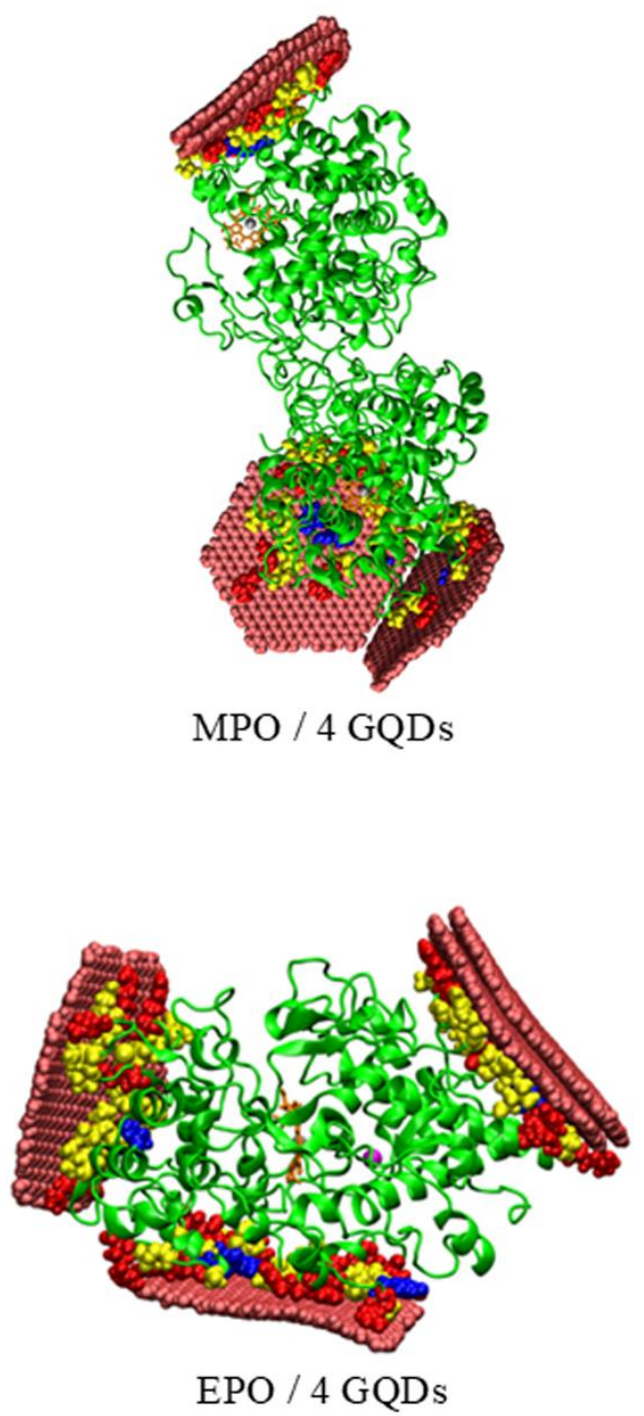

b
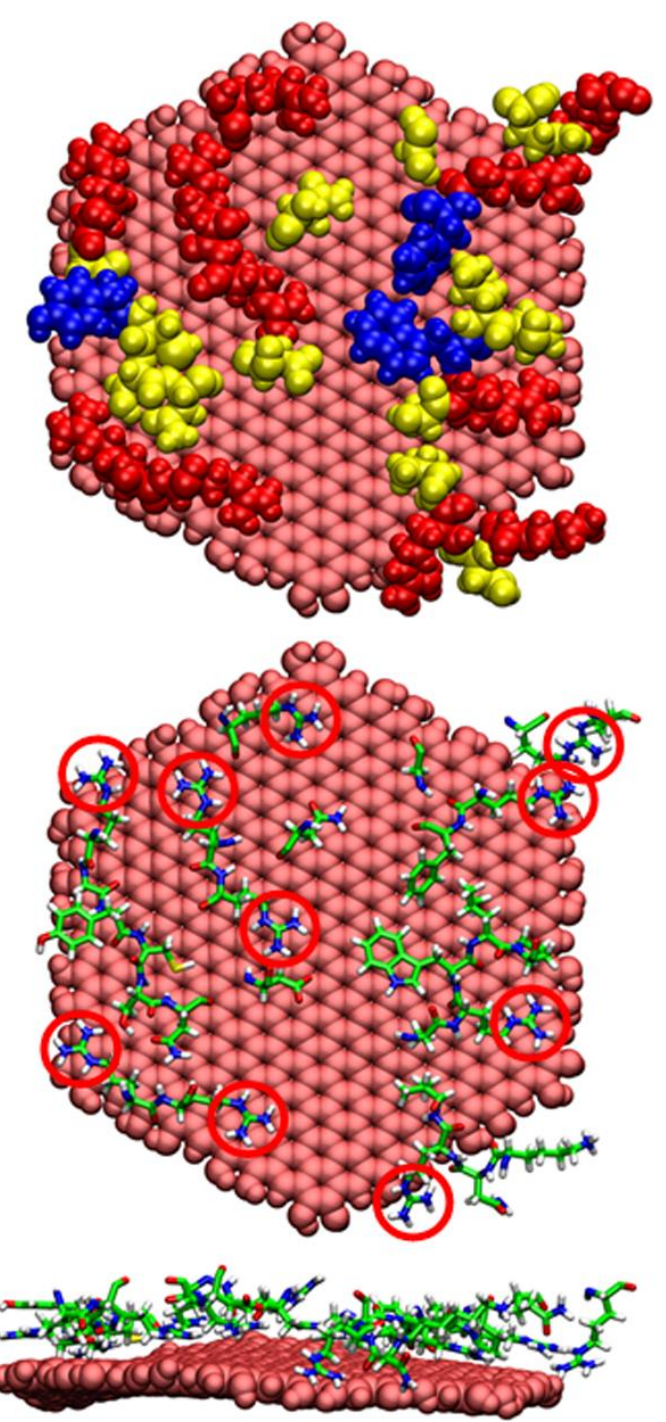

Figure 5. a) MD simulations of MPO and EPO interacting with the GQDs (van der Waals representation). In red positively charged residues (Arg, Lys, His), in blue aromatic residues (Phe, Trp, Tyr), and in yellow other amino acids. b) Final views of the interactions between one GQD and the amino acids in the EPO/4 GQD system (top). Final structures of the simulated systems with one GQD and the amino acids adsorbed on the surface (bottom). Red circles show the guanidine group of Arg. For clarity the hydroxyl groups are not shown at the edges of the GQDs. 